\title{
CONOCIMIENTO LOCAL Y USOS DE LA FAUNA SILVESTRE EN EL MUNICIPIO DE SAN ANTONIO DEL TEQUENDAMA (CUNDINAMARCA, COLOMBIA)
}

\section{LOCAL KNOWLEDGE AND WILDLIFE USES IN THE SAN ANTONIO DEL TEQUENDAMA MUNICIPALITY (CUNDINAMARCA, COLOMBIA)}

\begin{abstract}
Karin Osbahr ${ }^{1}$, Nicole Morales 2
${ }^{1}$ Líder Grupo de Investigación en Fauna Silvestre. Universidad de Ciencias Aplicadas y Ambientales U.D.C.A. Dirección para correspondencia: Calle 222 No. 55-37, Bogotá, D.C. E-mail: kosbahr@udca.edu.co 2 Investigadora Unidad de Investigación en Fauna Silvestre. Universidad de Ciencias Aplicadas y Ambientales U.D.C.A. Dirección para correspondencia: Calle 222 No. 55-37 Bogotá, D.C. E-mail: nicole.morales@javeriana.edu.co
\end{abstract}

Rev. U.D.C.A Act. \& Div. Cient. 15(1): 187- 197, 2012

\section{RESUMEN}

Tradicionalmente, los habitantes de la región andina han aprovechado los diferentes grupos de la fauna, tanto invertebrada como vertebrada; sin embargo, aunque la fauna en Colombia es bien conocida desde el punto de vista sistemático, existe un gran vacío de información en temas relacionados con los usos. El objetivo general de este estudio fue identificar la fauna silvestre conocida por los habitantes del municipio de San Antonio del Tequendama y evaluar la práctica que le dan desde sus saberes. A partir de encuestas aplicadas a la población rural y urbana, se encontraron seis tipos de aprovechamiento que involucran, especialmente, las clases Mammalia y Aves. La caza de subsistencia, el manejo como mascotas y las creencias o usos medicinales juegan un papel importante y son indicadores indispensables para fortalecer programas de conservación, que involucra a las comunidades locales.

Palabras clave: Índice de valor de uso, fauna silvestre, percepción cultural.

\section{SUMMARY}

Traditionally the inhabitants of the Andean region have taken advantage of the different groups of invertebrate and vertebrate fauna. However, although the fauna in Colombia is well known from the systematic point of view, there is a huge information gap on use related issues. The overall objective of this study was to identify the knowledge of the inhabitants of the municipality of San Antonio del Tequendama and evaluate how they use wildlife. From surveys of rural and urban population six types of use were identified that involve especially mammals and birds. Subsistence hunting, use as pets and beliefs or medicinal uses play an important role and are indicators to strengthen conservation programs that involve local communities.

Key words: Use value index, wildlife, cultural awareness.

\section{INTRODUCCIÓN}

El complejo eco-regional de los Andes sobresale por su topografía única con variedad de pisos térmicos y rangos altitudinales (Morello, 1984; Rodríguez et al. 2006), dando origen no solo a una composición florística compleja sino también a un alto endemismo y diversidad biológica (Myers et al. 2000; Tobón, 2009). Estudios paleontológicos han permitido reconocer que, a partir del intercambio faunístico entre América del Norte y del Sur, que tuvo lugar en épocas del Plioceno y del Pleistoceno, diversos linajes de mamíferos y de aves, como los carnívoros (Ursidae, Mustelidae, Felidae), tapires (Tapiridae), pecaríes y venados (Tayassuidae y Cervidae), ardillas (Sciuridae), conejos (Leporidae), tinamúes (Tinamidae), loros y afines (Psittacidae), colibríes (Trochilidae) y pavas (Cracidae), se integraron a la fauna regional (Webb, 1978; Reig, 1981). Según Correal \& van der Hammen (1977), a pesar de la variación climática y ambiental y de las actividades desarrolladas por el hombre primitivo, 
los abrigos rocosos de la región del Tequendama, en el Departamento de Cundinamarca, han proporcionado, a través del tiempo, las condiciones óptimas, para que animales silvestres de los órdenes Rodentia, Didelphimorphia, Cingulata, Carnivora, Lagomorpha y Cetartiodactyla permanezcan todavía en la región, aún cuando su abundancia haya podido cambiar positiva o negativamente, debido a los efectos causados por las actividades antrópicas actuales. Tradicionalmente, los habitantes de la región andina han hecho uso de esta biodiversidad, incluyendo los diferentes grupos de la fauna invertebrada y vertebrada. Algunas de estas prácticas, se remontan a la época prehispánica durante, la cual, la recolección de subproductos, como la miel de abejas silvestres, de la cacería y de la pesca eran una fuente de alimentación (Patiño, 1990). Las excavaciones arqueológicas indican que durante esta época los habitantes de la Sabana de Bogotá y sus alrededores cazaban las comadrejas (Mustela frenata), el borugo de páramo (Cuniculus taczanowskii), el coatí de montaña (Nasuella olivacea), el oso de anteojos (Tremarctos ornatus), la zarigüeya (Didelphis albiventris) y algunas aves, entre ellas las pavas de monte (Penelope sp.), patos, loros y palomas (Correal \& Pinto, 1983). Los lagos y los pantanos, a su vez, ofrecían una fuente de peces, como el capitán (Eremophilus mutisii), el capitán enano (Pygidium bogotense), los cangrejos sabaneros (Neostrengeria macropa) y algunos moluscos. Actualmente, muchas de estas prácticas han desaparecido, puesto que los bosques han sufrido una reducción del $74 \%$, debido, en su mayoría, a actividades antrópicas, que no sólo han impactado las coberturas vegetales y las fuentes hídricas sino, también, a las poblaciones de fauna silvestre que los habitan (MMA/DNP/ IAvH, 1995; FAO, 2001).

Aun cuando la fauna en Colombia es bien conocida desde el punto de vista sistemático, existe un gran vacío de información en temas relacionados con los usos, la valoración, el manejo y la conservación de la fauna silvestre, a nivel regional y local (Ojasti, 1993; 2000; Rubio-Torgler et al. 2000). Robinson \& Redford (1991) identificaron cinco tipos de uso de los recursos en el Neotrópico: subsistencia, mercados locales, comercio, cacería deportiva y cosecha o rancheo; sin embargo, es posible que de acuerdo a las características sociales, culturales o económicas propias de cada comunidad, sea posible identificar otros modos de empleo regionales o locales (Ojasti, 1993; Stearman, 2000). Por ende, la valoración cultural de la fauna, se basa en el saber popular generado, bien sea por el contacto directo con las diferentes especies o por los conocimientos tradicionales adquiridos a lo largo del tiempo. La comunidad conoce lo que es importante para ella y la definición de esa importancia es relativa y subjetiva, dependiendo de la experiencia, el conocimiento, las necesidades y hasta de la relación de los costos y beneficios tangibles (Londoño-Betancourth, 2009).
El objetivo general de este estudio fue identificar la fauna silvestre conocida por los habitantes del municipio de San Antonio del Tequendama y evaluar el uso que le dan a partir de sus saberes. Para ello, la investigación se enfocó en estimar la importancia de los mamíferos y las aves silvestres, que por su tamaño y colores suelen ser más llamativos para los habitantes; sin embargo, también se tuvieron en cuenta los anfibios, los reptiles y algunos invertebrados (Arachnida, Malacostraca e Insecta), pues son animales usualmente categorizados como peligrosos y, por lo mismo, pueden presentar, incluso, una mayor amenaza. La información obtenida en esta investigación será de utilidad para los programas de educación ambiental, que actualmente se desarrollan en la zona, en conjunto con la comunidad.

\section{MATERIALES Y MÉTODOS}

El municipio de San Antonio del Tequendama (Provincia del Tequendama) está situado en el centro occidente del Departamento de Cundinamarca (Colombia), a $4^{\circ} 37^{\prime} 08^{\prime \prime}$ de latitud Norte y $74^{\circ} 21^{\prime} 14^{\prime \prime}$ de longitud Oeste. Limita al occidente con el municipio de El Colegio; al Norte, con Tena y Bojacá; al oriente, con Soacha y al sur, con Granada. Su temperatura y su precipitación media anual son de $20,3^{\circ} \mathrm{C}$ y $1482 \mathrm{~mm}$, respectivamente. Se encuentra entre los 1.503 y $1.900 \mathrm{msnm}$, lo que le confiere un clima frío en zonas altas, y templado y cálido, en zonas bajas, permitiendo que su economía se desarrolle en torno a la ganadería y a la agricultura, especialmente, frutera, cafetera y de plantas ornamentales y aromáticas (IGAC, 1996; CGR-CDSS, 2010). Actualmente, el municipio tiene un área de 8944,76ha, representadas por dos áreas urbanas (64,59ha): la cabecera municipal San Antonio y la inspección de policía Santandercito; dos áreas suburbanas (44,07ha): Pueblo Nuevo y Pradilla-Los Naranjos y un área rural constituida, por veintiún veredas $(8828,24 \mathrm{ha})$ (IGAC, 1996).

Esta investigación tuvo lugar en el área urbana de Santandercito $(1.645 \mathrm{~m})$ y de San Antonio $(1.503 \mathrm{~m})$ y en las veredas Arracachal (sector Castalia y Pin), Quintas Colombia y Cusió (sector La Cadena), del área rural de San Antonio, en cercanías del Zoológico de Santacruz. La información recolectada fue primaria y cualitativa (Briones, 2003). Se aplicaron encuestas a 278 habitantes, entre los 14 y 95 años de edad, con un tiempo de permanencia en las áreas de estudio, de dos meses en adelante. Las encuestas incluyeron información sobre la persona encuestada e información sobre la fauna como: animales que conoce, animales que ha visto en la región, animales que ha dejado de ver en la región, usos y percepciones (¿Qué haría al encontrar un animal de monte? y ¿Cómo considera a los animales de monte: peligrosos, útiles, dañinos y/o inofensivos?). La información obtenida fue sistematizada en matrices (Excel 2003) y analizada con el 
método de análisis simple de la información. Por triangulación de fuentes (Geilfus, 1997), se obtuvo un listado potencial de especies de mamíferos y de aves, agrupadas por clase, orden y familia taxonómica, utilizando como base los nombres comunes mencionados por los pobladores y los datos de distribución registrados con respecto a la altitud de las áreas seleccionadas (1500-1800m), en Hilty \& Brown (1986), Emmons (1990), Alberico et al. (2000), Morales-Jiménez et al. (2004), Wilson \& Reeder, 2005, CI \& MAVDT (2006), UICN (2010), McMullan et al. (2010) y Remsen et al. (2011).

Los datos sobre la fauna que los pobladores han visto y han dejado de ver en la región, se analizaron por clase taxonómica con respecto al número total de registros (TR) y de encuestas (TE). Adicionalmente, se relacionó el número total de especies vistas o dejadas de ver y el tiempo de permanencia de los pobladores en la región. Para establecer la relación entre la fauna identificada y sus usos, se aplicó el Índice de Valor de Uso General (IVUis), que expresa la importancia o valor cultural de una especie determinada para todos los encuestados (Cotton, 1996). La fórmula aplicada fue:

$$
I U V_{i s}=\frac{\sum U_{i s}}{n}
$$

Donde: Uis es el número de usos mencionados por el encuestado; $i$, para la especie; $s \mathrm{y}, n$ es el número total de encuestados (Phillips \& Gentry, 1993).
Los registros obtenidos para usos se organizaron en matrices, articulando, mediante análisis de variables cruzadas y la prueba de Chi cuadrado $\left(X_{2}\right)$, el tipo de uso, en relación a la región encuestada, la fauna involucrada y la clase taxonómica. Específicamente, para el uso medicinal, se relacionó: enfermedad y fauna empleada, y para creencias: creencia y fauna involucrada. Finalmente, con respecto al tema de percepciones, se tuvieron en cuenta las acciones o las consideraciones mencionadas por los encuestados en cada región, con respecto al total de encuestas.

\section{RESULTADOS Y DISCUSIÓN}

Basado en los animales que la población mencionó haber visto en la región de San Antonio de Tequendama, se obtuvo un listado potencial, conformado por 89 especies de mamíferos, 51 terrestres y 38 voladoras, relacionadas a 50 nombres comunes y 90 especies de aves, relacionadas a 32 nombres comunes (Anexo 1). Los pobladores también mencionaron algunos reptiles (Clase Sauropsida), anfibios (Clase Amphibia) e Invertebrados (Clases Arachnida, Malacostraca e Insecta). La mayor frecuencia de registros calculada sobre el total de encuestas (TE) por localidad, se obtuvo para la Clase Mammalia (Tabla 1). En general, los pobladores de San Antonio y de Santandercito indicaron que la ardilla (Sciurus sp.), el borugo (Cuniculus sp.), la fara o runcho (Didelphis sp.), el armadillo (Dasypus sp.), la comadreja (Mustela sp.), el ñeque (Dasyprocta sp.), el oso perezoso (Choloepus sp.) y el conejo silvestre (Sylvilagus sp.) son vistos en la región

Tabla 1. Número de registros para cada clase taxonómica y proporciones con respecto al total de registros (TR) y al total de encuestas (TE) en cada zona visitada.

\begin{tabular}{|c|c|c|c|c|c|c|}
\hline \multicolumn{7}{|c|}{ ÁREAS DE ESTUDIO } \\
\hline \multirow[b]{2}{*}{$\begin{array}{c}\text { Clase } \\
\text { taxonómica }\end{array}$} & \multicolumn{3}{|c|}{ SAN ANTONIO } & \multicolumn{3}{|c|}{ SANTANDERCITO } \\
\hline & $\begin{array}{l}\text { Número de } \\
\text { registros }\end{array}$ & $\begin{array}{c}\text { \% registros } \\
(\mathrm{TR}=846)\end{array}$ & $\begin{array}{c}\text { Frecuencia } \\
(\mathrm{TE}=235)\end{array}$ & $\begin{array}{l}\text { Número de } \\
\text { registros }\end{array}$ & $\begin{array}{c}\text { \% registros } \\
(\mathrm{TR}=846)\end{array}$ & $\begin{array}{c}\text { Frecuencia } \\
(\mathrm{TE}=43)\end{array}$ \\
\hline Mammalia & 545 & 64,42 & 0,10 & 193 & 55,14 & 0,45 \\
\hline Aves & 136 & 16,08 & 0,02 & 100 & 28,57 & 0,22 \\
\hline Sauropsida & 117 & 13,83 & 0,04 & 46 & 13,14 & 0,19 \\
\hline Amphibia & 4 & 0,47 & 0,01 & 2 & 0,57 & 0,05 \\
\hline Arachnida & 37 & 4,37 & 0,03 & 9 & 2,57 & 0,09 \\
\hline Malacostraca & 6 & 0,71 & 0,03 & - & - & - \\
\hline Insecta & 1 & 0,12 & 0,004 & - & - & - \\
\hline
\end{tabular}


en mayor proporción que otros mamíferos, como el tigrillo (Felis sp.), el oso hormiguero (Tamandua sp.), el coatí (Nasuella olivacea), el perro de monte (Potos flavus), el venado (Mazama sp.) y el puercoespín (Coendou sp.). Por su parte, las aves más vistas en ambas regiones fueron la pava (Cracidae), las águilas (Accipitridae), el curruco o búho (Strigidae), los loros y pericos (Psittacidae), los canarios o chisgas (Fringillidae) y las palomas, tórtolas o torcazas (Columbidae); mientras que las menos vistos fueron la tingua (Rallidae), la guacharaca (Tinamidae), la gallineta (Cracidae), el pato silvestre (Anatidae), el tucán (Ramphastidae) y el golillon (especie no identificada).

El tiempo de permanencia en la región de los habitantes encuestados de ambos sexos osciló entre $<20(35,61 \%), 21$ 40 (32,74\%), 41-60 (17,63\%) hasta 60-81 (14,17\%) años. El rango de animales reportados mostró una tendencia indirectamente proporcional, de acuerdo a este tiempo de permanencia, donde el mayor porcentaje se centró entre cero a cinco especies diferentes. Únicamente, el rango entre seis a diez especies presentes fue reportado por todas las categorías de permanencia en la región (Figura 1a). Adicionalmente, el $71,94 \%$ de los encuestados ha dejado de ver entre una y tres especies, principalmente, de la Clase Mammalia, como Choloepus sp., Cuniculus sp. y Dasypus sp. El 5,40\% de los encuestados reportó entre cuatro y siete especies ausentes, mientras que el $22,66 \%$ asegura no extrañar a ninguna. Sobresale el hecho que los encuestados con más de 60 años de permanencia en la región indicaron la desaparición de hasta siete especies (Figura 1b). Es así como estos resultados muestran variaciones en la presencia y en la ausencia de especies faunísticas, a lo largo del tiempo. Aunque la aplicación de encuestas puede llevar a subestimaciones o sobreestimaciones de la riqueza y la abundancia, debido a posibles discrepancias entre las apreciaciones populares y las científicas (Fleck et al. 1999; Sánchez et al. 2004), la información aportada por los pobladores, que llevan diferentes periodos habitando la región, permite tener una visión histórica de la fauna local. Igualmente, ofrece información preliminar sobre el estado de conservación, partiendo de la percepción de los pobladores; sin embargo, es importante mencionar que durante el estudio se evidenció la influencia de la Fundación Zoológico Santacruz, puesto que durante las encuestas algunos pobladores mencionaron el jaguar (Panthera onca), cuya distribución no concuerda con el área de estudio.

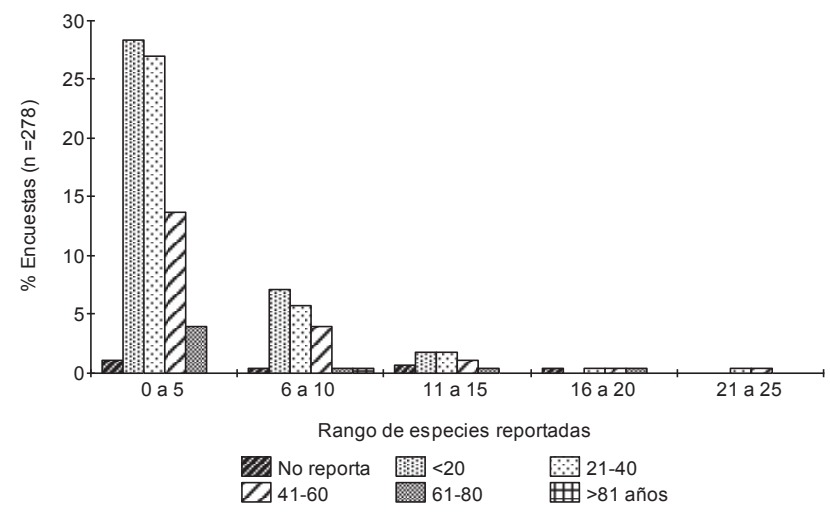

Figura 1b

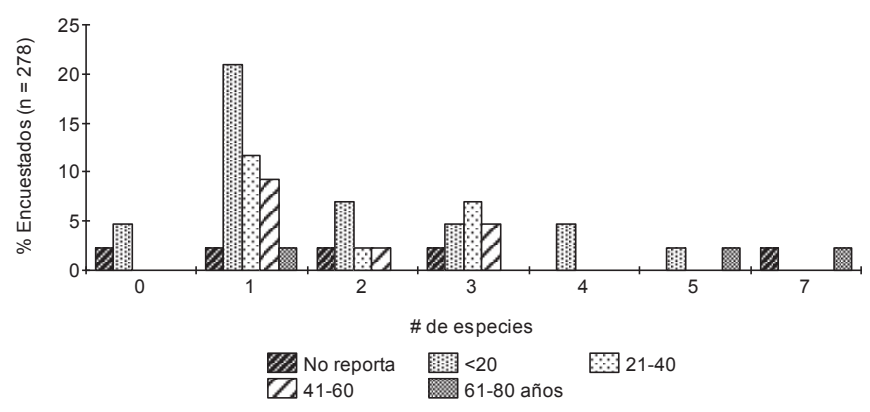

Figura 1. Relación entre el tiempo de permanencia en la región de San Antonio del Tequendama y número de especies reportadas. a) Número de especies presentes. b) Número de especies que han desaparecido en la zona. 
La población de esta región del Tequendama identificó cinco tipos de uso directo, la fauna silvestre (mascota, caza por alimento, caza por deporte, comercio y medicinal) y un uso indirecto, el cual, asocia las creencias. En general, los pobladores de San Antonio involucraron, en su mayoría, la utilización de la fauna como mascotas, usos medicinales y creencias (TR $=76.82 \%$, mientras que en Santandercito, el uso como mascotas, caza por alimento y creencias fue reportado en mayor proporción (TR $=76.06 \%$ ). Las seis categorías de uso identificadas registraron valores superiores para la población de la zona urbana (Figura 2a); sin embargo, la prueba de Chi cuadrado no registró diferencias estadísticamente significativas para las categorías de uso, muestreadas de acuerdo a la zona rural o urbana $\left(X_{2}=6,30 ; \mathrm{gl}=4 ; \mathrm{p}=0,177\right)$. Las Clases Mammalia y Aves fueron reportadas en cinco categorías de uso. En los mamíferos sobresale el aprovechamiento como alimento y mascotas, mientras que en las aves se reportaron mayor número de creencias y de usos medicinales (Figura 2b). Estas especies, con su respectivo Índice de Valor de Uso General, incluyen los géneros Sciurus sp. y Sylvilagus sp. (IUV = 0,06), los géneros Cebus sp., Choloepus sp. y las familias Cervidae y Canidae (IUV $=0,01$ ), Dasyprocta sp. (IUV $=0,05)$ y Didelphis sp. (IUV =0,04). El armadillo (Dasypus sp.) y el borugo (Cuniculus sp.) registraron el mayor Índice de Valor de Uso General (IUV = 0,15 y 0,10, respectivamente). En la Clase Aves, el uso se centró en las Familias Psittacidae (IUV = 0,06), Columbidae (IUV = 0,02) a la vez que en las familias Accipitridae, Turdidae, Trochilidae y Cuculidae (IUV =0,01). El mayor valor cultural, se obtuvo para la categoría de medicina y de creencias Strigidae (IUV $=0,17)$ y Cathartidae $(I U V=0,14)$. La clase Sauropsida, con dos reptiles relacionados (serpientes y tortuga morrocoy) es empleada como mascota y las clases Arachnida, Malacostraca e Insecta, aunque presentaron pocos registros referente a los usos, están relacionadas con la caza deportiva, la medicina y algunas creencias (Figura $2 b$ ). En general, la proporción de fauna empleada en los cinco tipos de uso es del 3,23\% (Dasypus sp.), en cuatro usos, del 6,45\% (Cuniculus sp.), en tres usos, del 22,58\% (Cathartidae), en dos usos, del 16,13\% (Strigidae) y, en un uso, del 51,61\%, indicando que, por lo general, las especies no están relacionadas con más de tres aspectos culturales de la comunidad.

Si bien es cierto que durante el presente estudio no se tuvo en cuenta el origen de la población encuestada es claro que el uso de la fauna se relaciona con procesos de colonización y con usos de la tierra, el cual, a su vez, se asocia con el origen y la costumbre de las poblaciones. De tal manera que el origen ancestral de la población urbana encuestada explicaría los resultados obtenidos que muestran que aún subsiste un fuerte arraigo hacia el uso tradicional de la fauna. En este contexto, la captura de mascotas sigue jugando un papel importante, involucrando, aunque en menor propor- ción, a mamíferos pequeños y medianos, a aves y a reptiles. Igualmente, los resultados indican que los habitantes de las zonas estudiadas en la región del Tequendama aún mantienen sus actividades de caza de subsistencia para la obtención de carne principalmente de Dasypus sp., Cuniculus sp., Dsyprocta sp., Sylvilagus sp., y Didelphis sp., lo cual, corrobora lo expuesto por Baptiste et al. (2002), quienes exponen que la región andina, contrario a las creencias, aún aporta un volumen considerable en el aprovechamiento de la fauna silvestre. Igualmente, se vislumbra que en la región del Tequendama la presión ejercida sobre las poblaciones de las especies coincide con aquellas para las que se registró un mayor número de categorías de uso por parte de las comunidades. A su vez, los resultados concuerdan con los reportes de uso de fauna en los departamentos de Boyacá (García et al. 2002), Cundinamarca (Vélez-Sosa, 2004) y Risaralda (Londoño-Betancourth, 2009).

Adicionalmente, la población aplica la caza deportiva y comercial. Según Ojasti (2000), la primera, al igual que la de subsistencia, está dirigida hacia el autoconsumo, mientras que la comercial aporta bienes de cambio y resalta el valor económico de la fauna (FAO/PNUMA, 1994). La caza comercial, como actividad (legal o ilegal), socialmente aceptada y altamente difundida en América Latina, presenta una diversidad de matices que dependen del producto ofrecido: la carne, las pieles, animales vivos, artesanías o elementos para la medicina popular, la industria farmacéutica y actividades culturales, relacionadas con creencias y con rituales (Cordero, 1990; TCA, 1995), por lo que la demanda de especies silvestres es cada vez más alta y suele involucrar diversos mamíferos, como los cérvidos (cuernos), los carnívoros (pieles, dientes, garras, mascotas), los roedores (pieles, mascotas) y los primates (garras, mascotas); aves, principalmente, Psittaciformes y Passeriformes (plumas, mascotas) y reptiles (pieles, caparazón, dientes, mascotas) (Cordero, 1990; Payan et al. 2007; Angulo et al. 2009; Palacios-Mosquera et al. 2010).

A nivel de uso medicinal y creencias, la información proporcionada por los pobladores permitió obtener características puntuales, adquiriendo datos similares a los obtenidos por García et al. (2002), en el Departamento de Boyacá. Se identificó que para prácticas medicinales, se emplea la sangre del armadillo para curar el asma, la anemia y el dolor de cabeza; el caldo de runcho, para las alergias; la grasa del zorro, para los dolores y la gastritis; la sangre de chulo, para curar el cáncer y la anemia y, el caldo de grillo, para poder orinar. En el caso de las creencias, se dice que cuando el curruco o el tres-pies canta es porque alguien va a morir; si canta una mirla u otro pájaro es porque va a llover o se acerca el invierno; si se sueña con culebras es porque va a llegar una visita indeseable; si un abejorro entra a la casa o una libélula o mariposa negra se estaciona en la casa o aparece de sorpresa 
Figura 2a

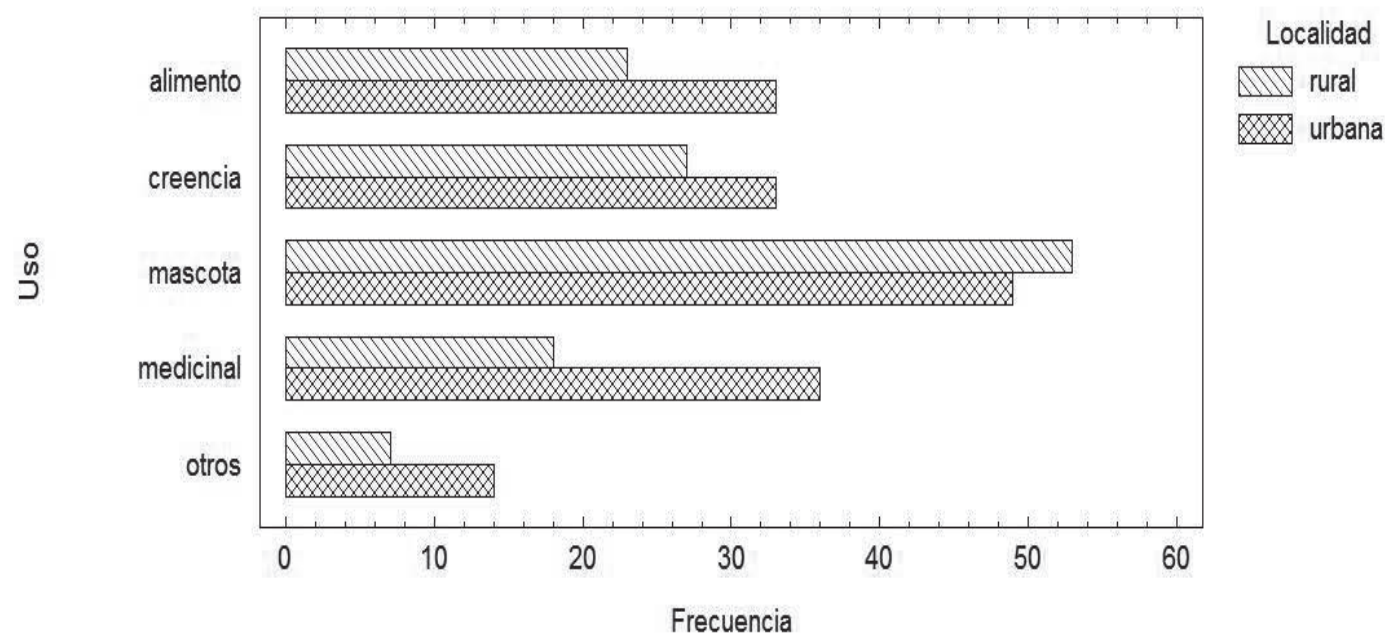

Figura $2 b$

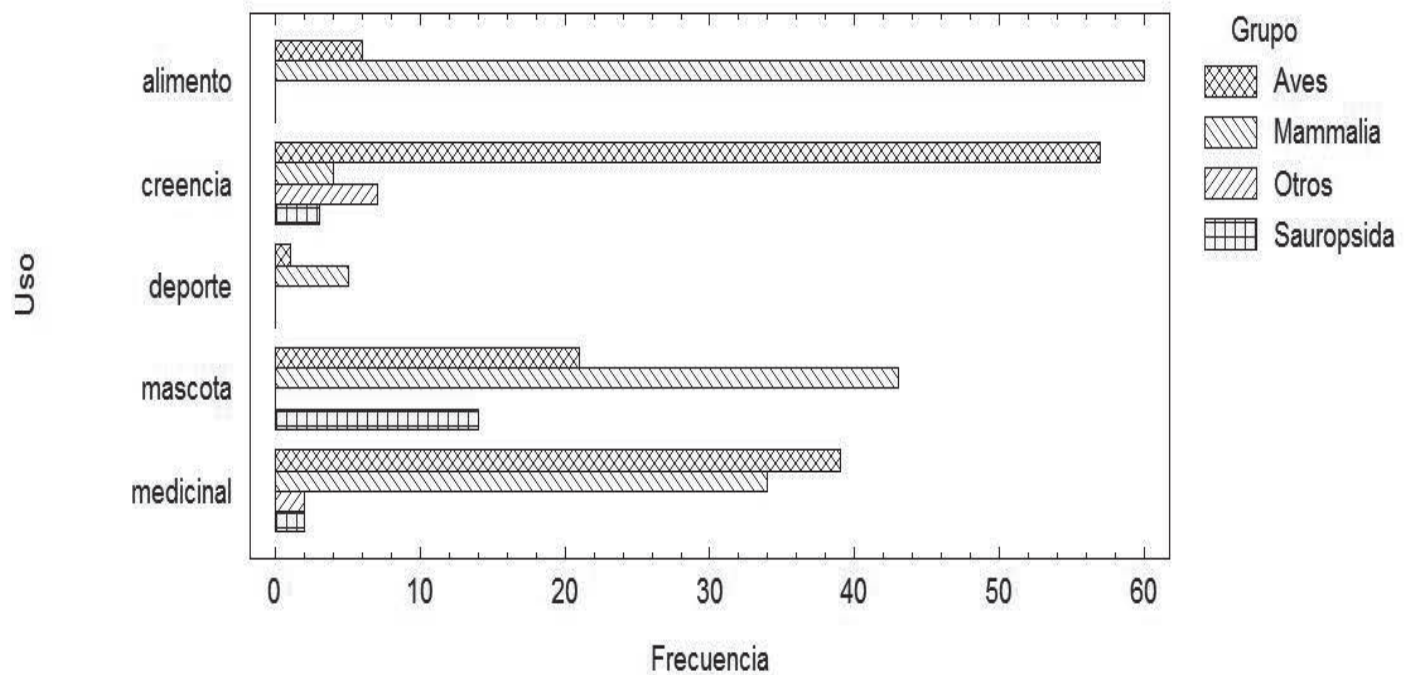

Figura 2. Categorías de uso de la fauna silvestre identificadas por los habitantes de la región de San Antonio del Tequendama. a) Distribución en zonas rurales y urbanas. b) Distribución de acuerdo al grupo taxonómico.

un colibrí o una araña es porque va a llegar visita. Raspar e ingerir los huesos del runcho es utilizado como afrodisiaco.

El estudio y la comprensión de estos tipos de uso a nivel local permiten tener una visión general de las especies de fauna silvestre presentes en la zona y una idea de la presión que la comunidad ejerce sobre las mismas. La utilidad de esta información en la planeación de estrategias de conservación y de uso sostenible de los recursos, a nivel local o regional, se basa en las percepciones o consideraciones que la población, desde su cultura, tiene respecto a la fauna, ya que como lo describe el concepto de valoración cultural, la percepción y el conocimiento empírico y tradicional que tienen los pobladores respecto al comportamiento, apariencia o forma de vida de un animal en particular, puede influir significativamente en la reacción que esta tenga ante su presencia (Robinson \& Redford, 1991; Ojasti, 2000; Vélez-Sosa, 2004); sin embargo, es importante tener en cuenta que las 
percepciones frente a la fauna silvestre pueden estar basadas en suposiciones y no en razones justificadas. Con base en esto, los pobladores de San Antonio y Santandercito perciben la fauna silvestre, en primera instancia, como inofensiva (64,39\%), aspecto que puede justificar el hecho que la mayoría de los encuestados hayan tenido animales silvestres como mascota; no obstante, al preguntarles qué harían al encontrar un animal de monte, el 73,02\% respondió que lo dejaría libre y sólo el 14,39\% respondió que lo usaría de mascota. Una explicación para estos resultados puede estar relacionada con los talleres de educación ambiental, que han surgido como iniciativas locales durante, los cuales, se recalca la importancia de conservar y de proteger las especies de fauna silvestre y su hábitat natural.

Para los pobladores de San Antonio, los animales de monte después de ser inofensivos son útiles, peligrosos y dañinos. Estas percepciones coinciden, a su vez, con las acciones mencionadas en las encuestas, pues en segundo lugar, ellos optarían por capturar el animal para mascota, lo llevarían al zoológico o lo matarían para alimento y, en tercer lugar, lo matarían si es venenoso, si los ataca o si se come a los animales domésticos. Sobresalen en este grupo, las especies de los Órdenes Rodentia y de las Familias Mustelidae y Didelphidae, mamíferos que encontraron en los asentamientos humanos una oportunidad de supervivencia y son consideradas como dañinas por entrar a los cultivos o comerse a las gallinas o como peligrosas, por su relación con la transmisión de enfermedades (Linares, 1976; López-Arévalo et al. 2002). Igualmente, al ser especies oportunistas son más abundantes alrededor de los asentamientos humanos, en comparación con otros lugares de bosque tropical, donde no hay asociación con el hombre. Otras especies involucradas en el conflicto humano-fauna silvestre son Tremarctos ornatus, Puma concolor, Leopardus sp. y Cerdocyon sp., mamíferos presentes en la Región del Tequendama. Por su tamaño y por sus características ecológicas y morfológicas entran a competir por espacio con las actividades antrópicas o pasan a ser consideradas piezas valiosas para el comercio, convirtiéndose en objetos de caza por parte de los habitantes, lo cual, explica, en gran medida, la disminución de sus poblaciones naturales (Vélez-Sosa, 2004; Payan et al. 2007; Angulo et al. 2009; Dickman, 2010).

Aún cuando durante el estudio no se evaluó la frecuencia de la cacería, por lo cual, posiblemente es ocasional, es claro que junto con el uso considerable de la fauna como mascotas en la zona urbana se viene ejerciendo una presión significativa sobre las poblaciones de aves y de mamíferos, por lo que se requiere de especial atención de estos temas en los programas de educación ambiental, que se desarrollan en San Antonio del Tequendama. Indudablemente, a largo plazo estas actividades, se reflejarán en el fortalecimiento de planes de conservación de la fauna silvestre.

Agradecimientos: Agradecemos a los estudiantes de la Facultad de Ciencias Pecuarias de la U.D.C.A Clara Marcela Rodríguez Moreno, Erika Milena Mayorga Torres, Marcela Castiblanco, Alejandro Cardozo, Jaime Buitrago, Daniel Mauricio Díaz Molano, Yeison Vera Vallejo y Daniel Bohórquez quienes aplicaron las encuestas en las áreas estudiadas; a los habitantes quienes muy cordialmente aportaron sus conocimientos; a Santiago Calvo, por la sistematización de la información y, finalmente, al Zoológico Santacruz, por facilitar el trabajo con los habitantes e incluir los resultados de la investigación en sus talleres de Educación Ambiental. Conflicto de intereses: El manuscrito fue preparado y revisado con la participación de todos los autores, quienes declaramos que no existe ningún conflicto de intereses que ponga en riesgo la validez de los resultados presentados.

\section{BIBLIOGRAFÍA}

1. ALBERICO, M.; CADENA, A.; HERNÁNDEZ-CAMACHO, J.; MUÑÓZ-SABA, Y. 2000. Mamíferos (Synapsida: Theria) de Colombia. Biota Colombiana. 1(1):43-75.

2. ANGULO, E.; DEVES, E.; JALMES, M.; COURCHAMP, F. 2009. Fatal attraction: rare species in the spotlight. Proc. Royal Soc. 276:1331-1337.

3. BAPTISTE-BALLERA, L.G.; HERNÁNDEZ-PÉREZ, S.; POLANCO-OCHOA, R.; QUICENO-MESA, M.P. 2002. La fauna silvestre colombiana: una historia económica y social de un proceso de marginalización. En: Ulloa, A. (ed.). Rostros culturales de la fauna. Instituto Colombiano de Antropología e Historia, Fundación Natura. p.295-340.

4. BRIONES, G. 2003. Métodos y técnicas de investigación para las ciencias sociales. Ed. Trillas (México). 364p.

5. CGR-CDSS. 2010. Sistema general de participaciones - Municipio de San Antonio del Tequendama. Contraloría General de la República. 34p.

6. CI - Conservación Internacional; MAVDT - Ministerio de Ambiente, Vivienda y Desarrollo Territorial. 2006. Libro rojo de los mamíferos de Colombia. Serie libros rojos de especies amenazadas de Colombia. Eds. Rodríguez-Mahecha, J.V., Alberico, M., Trujillo, F. \& Jorgenson, J. (Bogotá, Colombia). 433p. 
7. CORDERO, R. 1990. Aprovechamiento de la fauna silvestre en Barlovento, Estado Miranda, Venezuela. Vida Silv. Neotrop. 2(2):70-74.

8. CORREAL, U.G.; van der HAMMEN. 1977. Investigaciones arqueológicas en los abrigos rocosos del Tequendama. Ed. Fondo de promoción de la cultura del Banco Popular (Bogotá, Colombia). 194p.

9. CORREAL, G.; PINTO, M. 1983. Investigaciones arqueológicas en el municipio de Zipacón, Cundinamarca. Fundación de Investigaciones Arqueológicas Nacionales, Banco de la República. 58p.

10. COTTON, C. 1996. Ethnobotany. Principles and Applications. Ed. John Wiley \& Sons (Neww York, U.S.A). 424p.

11. DICKMAN, A.J. 2010. Complexities of conflict: the importance of considering social factors for effectively resolving human-wildlife conflict. Animal Conserv. 13:458-466.

12. EMMONS, L. 1990. Neotropical Rainforest Mammals, A Field Guide. Ed. The University of Chicago Press (London). 281p.

13. FAO. 2001. Causas y tendencias de la deforestación en América Latina. Documento de trabajo 52. Programa de Evaluación de Recursos Forestales (Roma). 91p.

14. FAO/PNUMA. 1994. Estado de conservación de la fauna Silvestre del Cono Sur Sudamericano. Oficina Regional de la FAO para América Latina y el Caribe (Santiago, Chile). 120p.

15. FLECK, D.W.; VOSS, R.S.; PATTON, J.L. 1999. Biological basis of saki (Pithecia) folk species recognized by the Matses Indians of Amazonian Perú. Int.l J. Primat. 20:1005-1008.

16. HILTY, S.L.; BROWN, B. 1986. A guide to the birds of Colombia. Princeton University Press. 836p.

17. GARCÍA, G.A.; PERICO, D.; ROCHA, C. 2002. Uso de la fauna silvestre en los alrededores del cerro de Mamapacha (Boyacá-Colombia). En: Durán, C.; Castaño, U. (Eds Páramos. Memorias Congreso Mundial de Páramos. Editorial Gente Nueva, p.887-899.

18. GEILFUS, F. 1997. 80 herramientas para el desarrollo participativo: diagnóstico, planificación, monitoreo, evaluación. GTZ, IICA-Holanda Laderas. 293p.
19. IGAC. 1996. Diccionario geográfico de Colombia. Tomo III. Ed. IGAC (Bogotá, Colombia). 615p.

20. LINARES, O.F. 1976. "Garden hunting" in the American tropics. Human Ecol. 4:331-349.

21. LONDOÑO-BETANCOURTH, J.C. 2009. Valoración cultural del uso e importancia de la fauna silvestre en cautividad en tres barrios de Pereira (Risaralda). Bol. Cient. Museo Hist. Nat. 13(1):33-46.

22. LÓPEZ-ARÉVALO, H.F.; MORALES-JIMÉNEZ, A.L.; MATALLAN, C. 2002. Aproximación a los efectos de las actividades antrópicas sobre la fauna de vertebrados del páramo Colombiano. Durán, C.; Castaño, U. (Eds.). Congreso Mundial Páramos. Ed. Gente Nueva. p.465-479.

23. MMA - Ministerio del Medio Ambiente; DNP - Departamento de Planeación Nacional; IAvH -Instituto Alexander von Humboldt. 1995. Política Nacional de Biodiversidad. 34p.

24. McMULLAN, M.; DONEGAN, T.; QUEVEDO, A. 2010. Field guide to the birds of Colombia. Fundación ProAves. 228p.

25. MORALES-JIMÉNEZ, A.L.; SÁNCHEZ, F.; POVEDA, K.; CADENA, A. 2004. Mamíferos terrestres y voladores de Colombia, Guía de campo. Ed. Ramos López (Bogotá, Colombia). 248p.

26. MORELLO, J. 1984. Perfil ecológico de Sudamérica. Ed. Cultura Hispánica (Barcelona, España). 96p.

27. MYERS, N.M.; MITTERMEIER, R.; da FONSECA, G.; KENT, J. 2000. Biodiversity hotspots for conservation priorities. Nature 403: 853-858.

28. OJASTI, J. 2000. Manejo de Fauna Silvestre Neotropical. En: Dallmeier, F. (ed.). SIMAB Series (5). Smithsonian Inst./MAB Program (Washington D.C). p.73-106.

29. OJASTI, J. 1993. Utilización de la fauna silvestre en América Latina. Situación y perspectivas para un manejo sostenible. Vol. 25. Ed. Food \& Agricultura Org. (Roma, Italia). 248p.

30. PALACIOS-MOSQUERA, L.; MENA-ROJAS, O.P.; SÁNCHEZ-LOZANO, L.E. 2010. Uso tradicional de osos perezosos (Bradypus variegatus y Choloepus hoffmanni) en seis municipios del departamento del Chocó, Colombia. Bioetnia. 7(1):4-9. 
31. PATIÑO, V.M. 1990. Alimentos indígenas de origen animal. En: Historia de la cultura material en América equinoccial. Tomo I. Alimentación y alimentos. Instituto Caro y Cuervo, Biblioteca Ezequiel Uricochea, Bogotá. p.120-163.

32. PAYAN, E.; MESA, M.P.Q.; FRANCO, A.M. 2007. Los felinos como especies focales y de alto valor cultural. Serie especies colombianas 7. Instituto de Investigación de Recursos Biológicos Alexander von Humboldt. $15 p$.

33. PHILLIPS, O.; GENTRY, A. 1993. The useful plants of Tambopata, Perú: I. Statistical Hypothesis test with a new quantitative technique. Soc. Econom. Bot. 47(1):15-32.

34. REIG, O.A. 1981. Teoría del origen y desarrollo de la fauna de mamíferos de América del Sur. Nature (Mar del Plata) 1:1-162.

35. REMSEN, J.V. Jr.; CADENA, C.D.; JARAMILLO, A.; NORES, M.; PACHECO, J.F.; PÉREZ-EMÁN, J.; ROBBINS, M.B.; STILES, F.G.; STOTZ, D.F.; ZIMMER, K.J. 2011. A classification of the bird species of South America. American Ornithologists' Union. Version 8. Disponible desde Internet en http:/www.museum. lsu.edu/ Remsen/SACCBaseline.html (con acceso el 16/08/2011).

36. ROBINSON, J.; REDFORD, K.H. 1991. Neotropical Wildlife use and conservation. Ed. The University of Chicago Press (Londres). 520p.

37. RODRÍGUIEZ, N.; ARMENTERAS, D.; MORALES, M.; ROMERO, M. 2006. Ecosistemas de los Andes colombianos. 2da. Ed. Instituto de investigación de Recursos Biológicos Alexander Von Humboldt (Bogotá, Colombia). 154p.

38. RUBIO-TORGLER, H.; ULLOA, A.; CAMPOS-ROZO, C. 2000. Manejo de la fauna de caza, una construcción a partir de lo local. Fundación Natura, UASPNN, OEI, ICANH, WWF Bogotá. 160p.
39. SÁNCHEZ, F.; SÁNCHEZ-PALOMINO, P.; CADENA, A. 2004. Inventario de mamíferos en un bosque de los Andes Centrales de Colombia. Caldasia 26(1):291309.

40. STEARMAN, A.M. 2000. A pound of flesh: Social change and modernization as factors in hunting sustainability among Neotropical indigenous societies. En: Robinson, J.G.;Bennett, E.L. (eds). Hunting for sustainability in tropical forests. Ed. Columbia University Press (New York), p.233-250.

41. TCA. 1995. Uso y conservación de la fauna silvestre en la Amazonia. Tratado de Cooperación Amazónica, Secretaría Pro-Tempore (Lima). 216p.

42. TOBÓN, C. 2009. Los bosques andinos y el agua. Serie investigación y sistematización \#4. Ed. ECOBONA (Quito). 64p.

43. UICN. 2010. IUCN Red List of Threatened Species. Version 2010.4. Disponible desde Internet en: http://www. iucnredlist.org (con acceso 16/03/2011).

44. VÉLEZ-SOSA, M. 2004. Diagnóstico del uso de fauna silvestre en las veredas Mundo Nuevo, el Manzano y la Jangada en la reserva forestal protectora de los ríos Blanco y Negro en el municipio de La Calera, Cundinamarca, Colombia. Memorias Congreso de Manejo de Fauna Silvestre en Amazonia y Latinoamérica. p.330-335.

45. WEBB, S.D. 1978. A history of savanna vertebrates in the New World. Part II South America and the great interchange. Ann. Rev. Ecol. Syst. 9:393-426.

46. WILSON, D.E.; REEDER, D.M. 2005. Mammal species of the World. Third Edition. The John Hopkins University Press. 1945p.

Recibido: Junio 19 de 2011

Aceptado: Enero 25 de 2012 


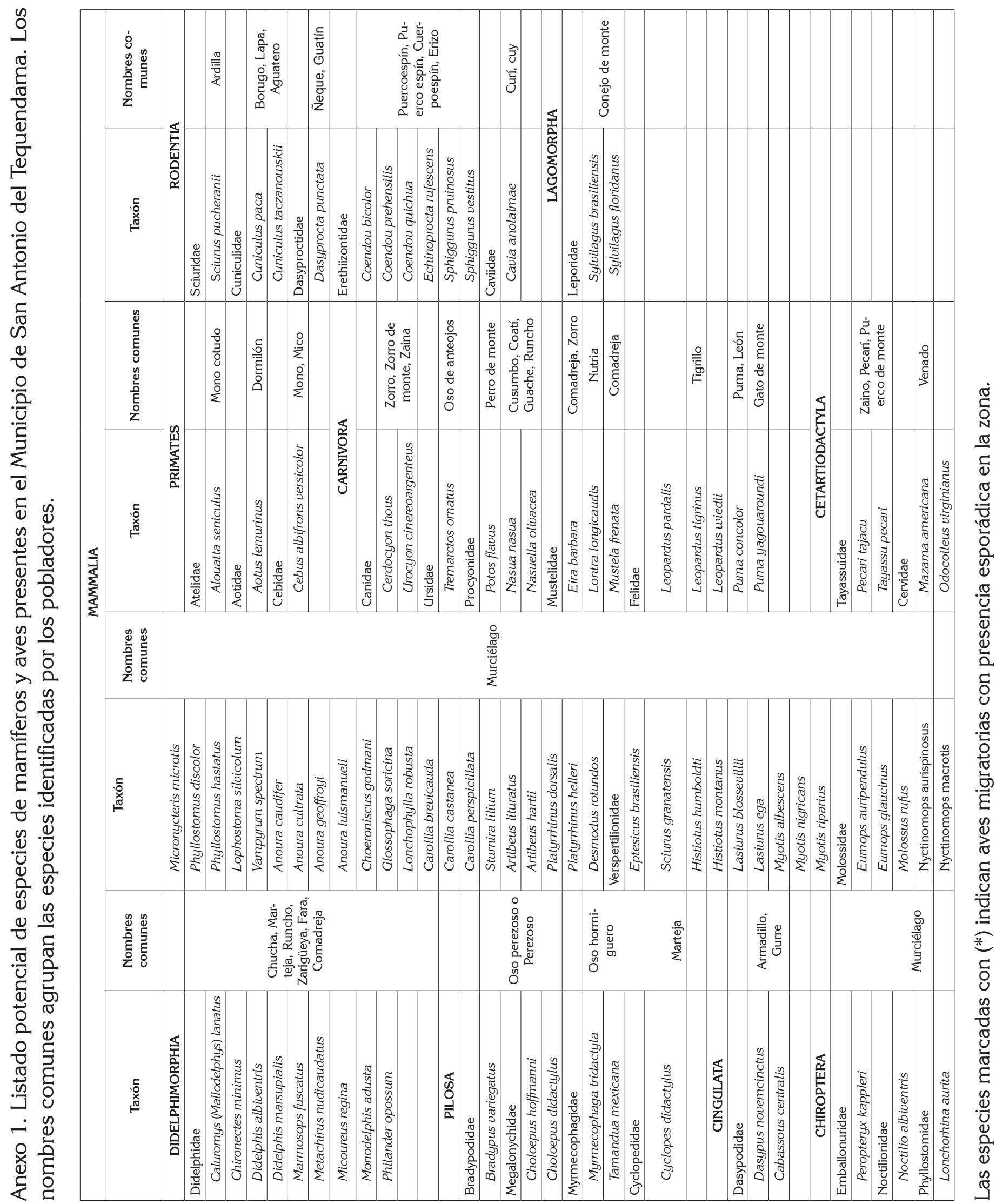




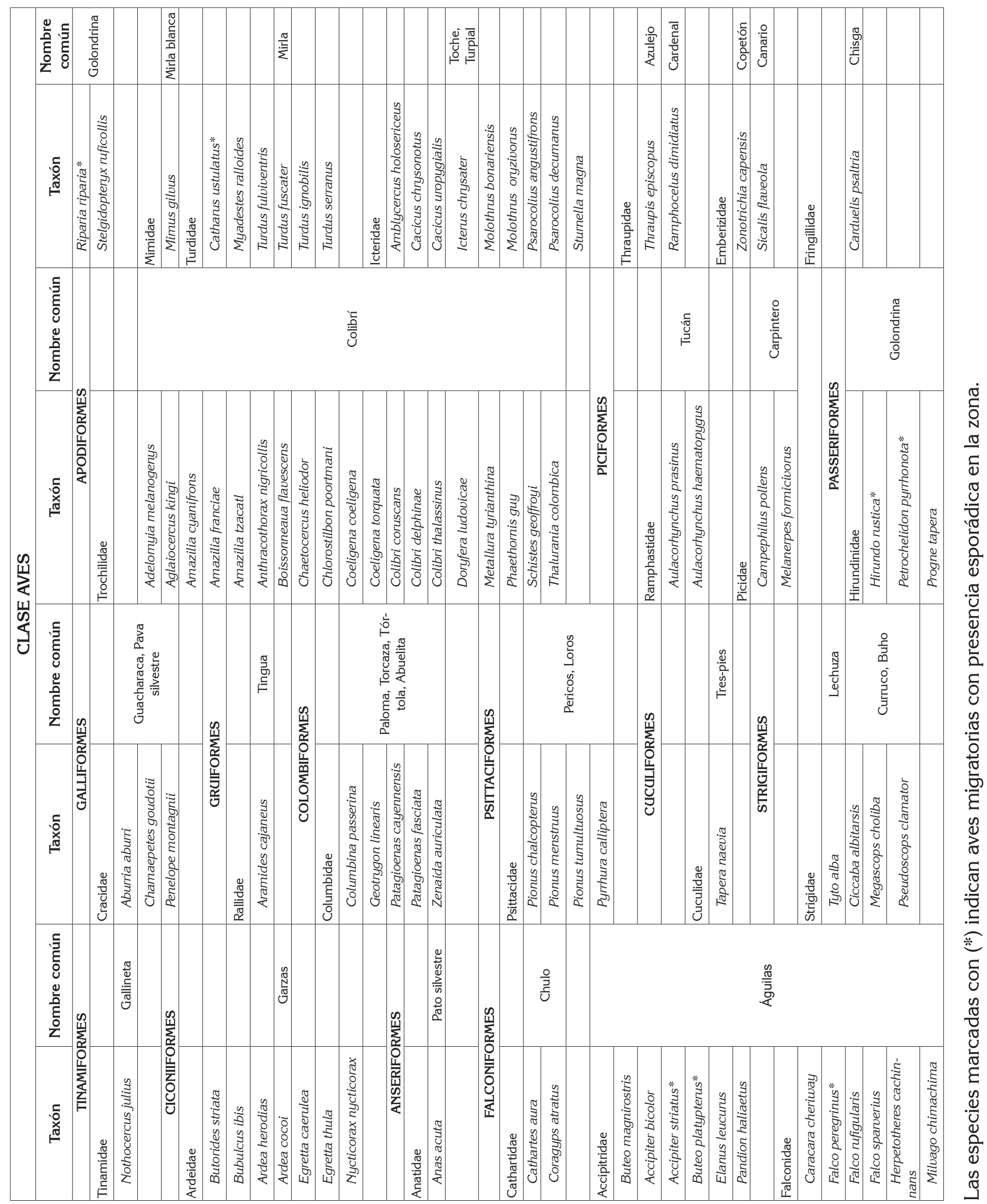

